\title{
INTEGRATION OF REGIONAL AND ASSET SATELLITE OBSERVATIONS FOR ASSESSMENT OF INFRASTRUCTURE RESILIENCE
}

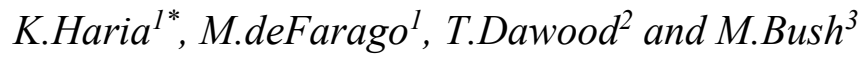 \\ ${ }^{1}$ Telespazio VEGA UK, Luton, UK \\ ${ }^{2}$ EDF Energy, London, UK \\ ${ }^{3}$ Transport for London, London, UK \\ * Corresponding author
}

\begin{abstract}
Satellite Structural Health Monitoring (S-SHM ${ }^{\mathrm{TM}}$ ) is an innovative approach that utilises satellite derived measurements (Interferometric Synthetic Aperture Radar (InSAR) analysis) of displacement with 3D Building Information Model (BIM) and expands the utility of InSAR beyond its conventional monitoring application. This new capability is centred on development of early warning and prediction of structural deformation by incorporating InSAR into structural stability algorithms. This will allow the asset condition to be highlighted, thus aiding decision making for multiple asset management.
\end{abstract}

Assets studied in isolation give an incomplete view of resilience. It is only when asset condition analysis is integrated with its environment that the full dynamics of the infrastructure come to life, providing improvements for infrastructure resilience assessment. Good resilience assessments should have the ability to reduce the magnitude and/or duration of disruptive events and to improve the ability to anticipate, adapt or rapidly recover from a potentially disruptive event. Hence, there exists a need for a harmonized resilience assessment methodology that is able to reduce the impact and the consequences for short, medium and long-term events affecting infrastructures.

This paper presents the concept of S-SHM ${ }^{\mathrm{TM}}$ and its process to derive a timeline of results, showing the health of the assets and surrounding regions. The paper also presents the outcomes of S-SHM ${ }^{\mathrm{TM}}$ for two case studies, showing a real promise of this approach for remote asset surveillance. Finally, the paper presents a summary of further implementations of S-SHM ${ }^{\mathrm{TM}}$, which aim to enhance its operability and therefore the value to the end-users.

\section{Introduction}

Asset managers require reliable \& long-term monitoring and assessment of their asset performance and condition so that its operation and utilisation can be maximised and maintained throughout its design lifetime. This requires that maintenance of any component of the asset that might otherwise cause the asset to operate sub-optimally be scheduled at appropriate times prior to structural failures. Such an early warning indicator of structural health is based on the concept of preventive maintenance, which is typically performed while the asset is still operational and to ensure that it does not unexpectedly fail or fall below required performance standards. Preventive maintenance is the first line of defence against unplanned downtime and equipment failures and empirically has been shown to reduce the need for - and higher cost of - capital equipment replacement by as much as 3 to 9 times less (O'Brien, 2017) than emergency or unplanned maintenance. For critical assets, such as power stations, transport hubs, airports, harbours, etc., downtime implies service denial for customers and dependent services, which therefore not only needs to be kept to a minimum, but preferably eliminated altogether. The process of planned maintenance makes tasks more efficient and also removes the effect of maintenance on the operations of the facility.

Key to preventive maintenance is the application of Structural Health Monitoring (SHM) techniques providing a level of prediction (through early warning) and hence increased accuracy of failure timing over conventional routine survey methods.

\section{SHM and Challenges}

SHM is a process of monitoring the health of structural assets over a period of time using an array of sensors. SHM data normalisation is the process of separating changes in sensor output caused by damage and changes caused by varying environmental conditions. Since most SHM systems do not continuously monitor, it is difficult to normalize the dataespecially when point sensors are used to collect the information (Shawn, 2005; Tongue).

- Many structural health monitoring systems rely on point sensors, to monitor assets. The limitations of point sensors are not about accuracy or reliability, rather it is about insight. Events that occur between 
critical points can be missed leading to loss of structural information.

- Issues in relation to sensor integration, calibration, reliability and effect of environmental conditions can have a direct impact on the quality of structural health data.

Installing in-situ structural health monitoring systems in various structures is a complex and cumbersome process due to the fact that the size and shape of structures vary significantly and the access to those structures can also be difficult. A single structural health monitoring system is not suited for the entire range of applications and differs based on materials, components, geometrics and damage scenarios. Sensors also need to be robust, accurate, reliable and durable, and any damage or failure to the sensor could impact the purpose of the whole system.

\section{Satellite-Structural Health Monitoring (S- SHM $^{\mathrm{TM}}$ )}

The Satellite-Structural Health Monitoring (S-SHM $\left.{ }^{\mathrm{TM}}\right)$ concept is a combination of satellite surveying and structural health monitoring. S-SHM ${ }^{\mathrm{TM}}$ is an innovative new structural health monitoring approach that utilises satellite-derived measurements (Interferometric Synthetic Aperture Radar (InSAR)) of displacement with 3D Building Information Model (BIM) modelling of the structure.

InSAR alone is a useful source of data for assisting asset condition assessment in a $2 \mathrm{D}$ environment to aid the management and prioritisation of site visits, in-situ monitoring and ultimately interventions to structures forming Critical National Infrastructure (CNI). This has been applied to flood defences along the Thames Estuary in the UK whereby InSAR measurements have been used to understand the rate of movement of individual assets over time. This offers the possibility to prioritise specific assets for remedial maintenance work based on the rate of subsidence or uplift and the condition assessment report.

S-SHM ${ }^{\mathrm{TM}}$ takes the analysis beyond the $2 \mathrm{D}$ environment by incorporating InSAR into structural stability algorithms that allow asset condition to be highlighted thus aiding asset management for multiple assets, which is an extremely powerful transformational capability for asset managers of large estates. The combination of satellite with BIM and structural modelling ensures that the satellite derived data can be directly actionable - the results informs the health of the structure as opposed to the current situation that only provides information on displacement. S-SHM ${ }^{\mathrm{TM}}$ can achieve a lower per-asset cost by sensing from the satellite rather than placing in-situ sensors on the asset. However, the integration of measurements from other surveying systems along with InSAR will increase the richness of the assessment on asset health.

\section{S-SHM ${ }^{\mathrm{TM}}$ Process}

The S-SHM ${ }^{\mathrm{TM}}$ process combines InSAR data with 3D model information by means of feature identifiers and classifying InSAR data into customizable groups and scenarios. This allows a series of structural health tests to be performed. Warnings are raised when specific PS measurements exceed customized thresholds.

$\mathrm{S}-\mathrm{SHM}^{\mathrm{TM}}$ process is designed around three interconnected layers, see Figure 1:

Figure 1: S-SHM ${ }^{\mathrm{TM}}$ Data Layers

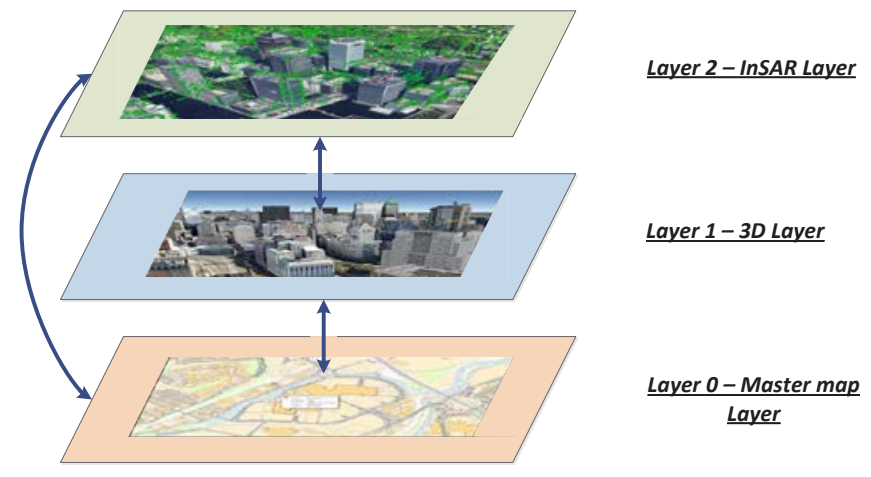

- Layer 0 Master Map or Topographic Map Layer This layer refers to topographical measurements over the Area of Interest (AOI). This layer enables the user to assess and configure topographic measurements for various landmarks over the AOI. These measurements include information such as coordinates of specific points, how they relate to $3 \mathrm{D}$ models and information on terrain for the points or the structure.

- Layer 1 3D Layer - This layer refers to 3D or a Building Information Model (BIM) of the asset being monitored and its surrounding areas. 3D models need to be of sufficient detail so that InSAR data can be overlaid on them. Modern constructions may have been built from 3D models however older buildings such as bridges, train stations etc. generally do not have digital building information. Where 3D models are not available, models need to be prepared with sufficient detail.

- Layer 2 InSAR Layer - This layer refers to the outputs of Interferometric analysis performed on SAR data. The InSAR analysis provides a cloud of geolocated displacement measurement points over the AOI. In order to perform InSAR analysis, SAR data over the AOI needs to be captured over a period of time (nominally 1 year) in order to establish a baseline. Throughout the monitoring period, data is acquired and processed for routine monitoring operations. 
$\mathrm{S}^{-\mathrm{SHM}^{\mathrm{TM}}}{ }^{\mathrm{TM}}$ process is split into four phases as shown in Figure 2:

Figure 2: S-SHM ${ }^{\mathrm{TM}}$ Process Phases

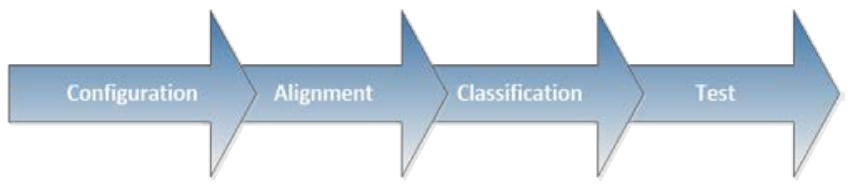

These phases are described in subsequent sections.

\subsection{Configuration Phase}

In this phase, all input data is gathered and configured. The input data mainly consists of:

- Information about the assets and surrounding areas from the Asset manager. This may include some of the following: information on the current monitoring process; list of failures; threshold values of acceptable movement or deformation for the structure and its components; geological data; thermal data; stress related data; structural data such as Building Information Models (BIM) or building plans and any other information which can affect structural monitoring.

- If a 3D BIM is not available then that needs to be produced using asset data.

- Topographic Maps (TM) data over the Area Of Interest (AOI) is acquired in this phase.

- Satellite imagery over the AOI may be acquired before and/or during this phase in order to establish the InSAR baseline.

The gathered input data is ingested into a relational database. Configuration of 3D models is performed using 3D modelling software and $\mathrm{S}-\mathrm{SHM}^{\mathrm{TM}}$ software. The process involves deriving a uniformly distributed set of points belonging to the $3 \mathrm{D}$ model. The software allows storage of $3 \mathrm{D}$ models in a database in the form of vertices and faces and their relationship. The scripts also create a cloud of points based on the ingested geometry using interpolation.

Configuration of InSAR data is performed by importing these datasets in which the information on Persistent Scatterers (PS). Topographic heights on buildings are ingested on top of the topographic areas imported in this format. Each of the InSAR PS has a unique identifier and a list of measurements of motion over time. These measurements are referred to as the displacement along the Line of Sight ( $\mathrm{LoS})$ of the satellite.

Configuration of TM is performed on the following layers:

- Vector 2D Areas, Lines and Point of interest;

- Raster Terrain Heights;

- Building Heights on top of vector 2D areas.

Configuration of Group of Features involves the creation of groups for:

- Geometric Faces;

- $\quad$ TM features;

- Settlement Information.

The Group of Features is stored in a database.

\subsection{Alignment Phase}

In this phase, alignment is performed between 3D model geometric data sets, InSAR data sets and topographic data sets.

Alignment between the $3 \mathrm{D}$ model and InSAR is performed by configuring a registered set of points. A registered point is basically mapping between a point in 3D model/InSAR data set and the corresponding TM data point.

Figure 3: InSAR data set as a cloud of points overlaying on top of the 3D model

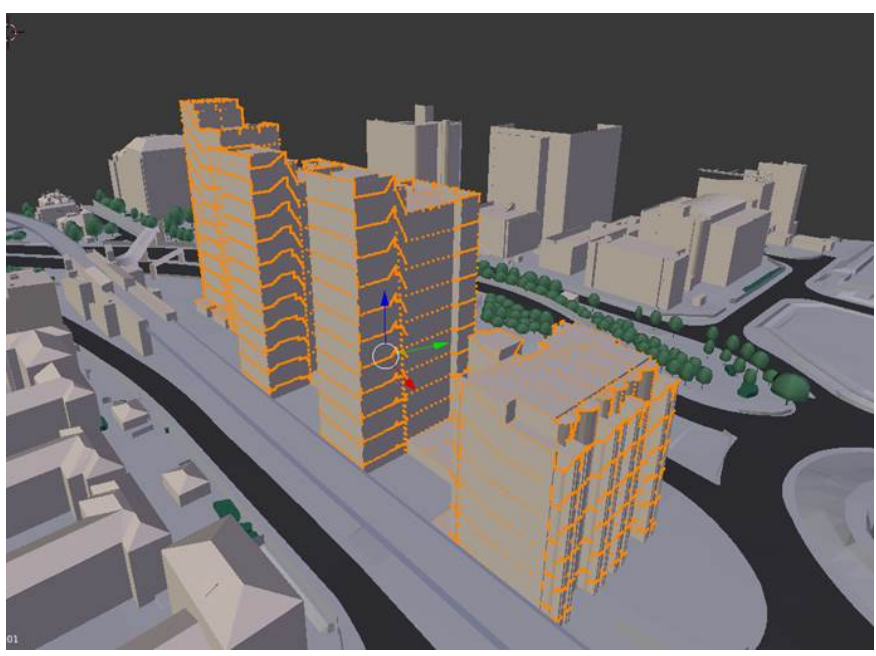

Alignment between InSAR data and TM features allows assignment of TM Topographic feature to each InSAR point. This categorises the InSAR points and enables proper classification of TM categories. 
Figure 4: InSAR Data Set on top of TM Topographic Lines

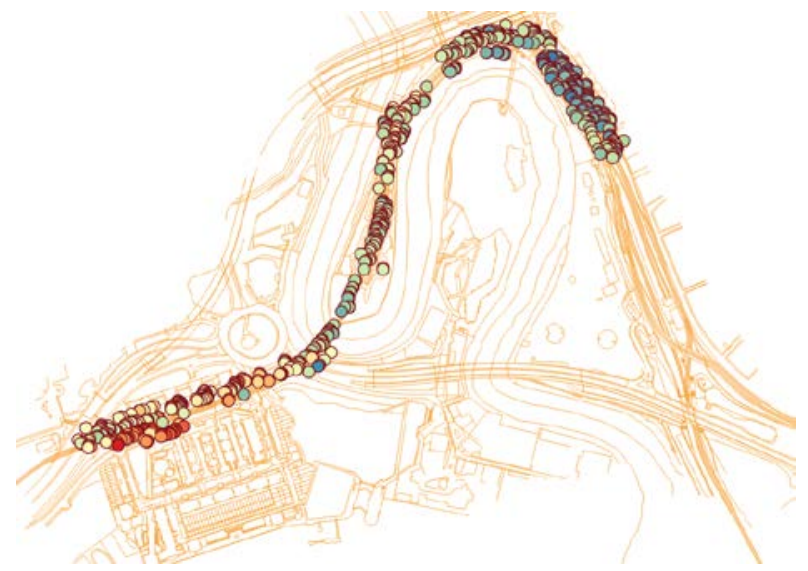

\subsection{Classification Phase}

In this phase classifications of InSAR data points with respect to a Group of Features take place. These Groups of Features are formed by 3D model and TM features which are customizable from using python scripts.

\subsection{Test Phase}

In this phase the classified data is put through the pre-defined tests such as Line of Sight (LoS) displacement analysis, time series analysis or other complex tests. Tests can be related to health condition, routine monitoring but also for prediction of structural health. The outputs are presented in a format that can be viewed in 3D visualisation capability. The set of tests can be defined after consultation with the user.

To perform a test on each InSAR point of its LoS displacement, RAG (Red, Amber, Green) thresholds are defined. When the absolute value of a PS measurement on a particular date exceeds the threshold assigned to the RAG then the InSAR is flagged. This is the basic test on $\mathrm{LoS}$ displacements within a scenario.

Additional tests can be developed and added to the software. A list of potential tests is provided in section 5 .

\section{S-SHM ${ }^{\mathrm{TM}}$ Results and Conclusions}

The S-SHM ${ }^{\mathrm{TM}}$ process has been executed for the following test cases for current and historical time durations:

- TfL site - East India \&Canning Town stations, the connecting railway track and surrounding regions

- Historical InSAR ground deformation data from 2011-2015 was analysed

- EDF site - Hinkley Point B nuclear power station

- InSAR data was generated from CosmoSkymed Stripmap and Spotlight data acquired between November 2016 and November 2017.
With regards to the TfL case study, outputs were promising and provided an independent validation of S-SHM ${ }^{\mathrm{TM}}$ concept. The Amber and Red thresholds were exceeded between October 2013 and May 2014. This is shown below in Figure 5 and Figure 6. A TfL assessment report identified surface ground movement greater than predicted from the indicative scheme. This was due to dewatering between late 2013 and early 2014 in vicinity of the River Lea. It was detected using in-situ sensors and had raised red triggers around the area.

Figure 5: Measurements on 17th October 2013 showing mostly Green values (normal values or stability).

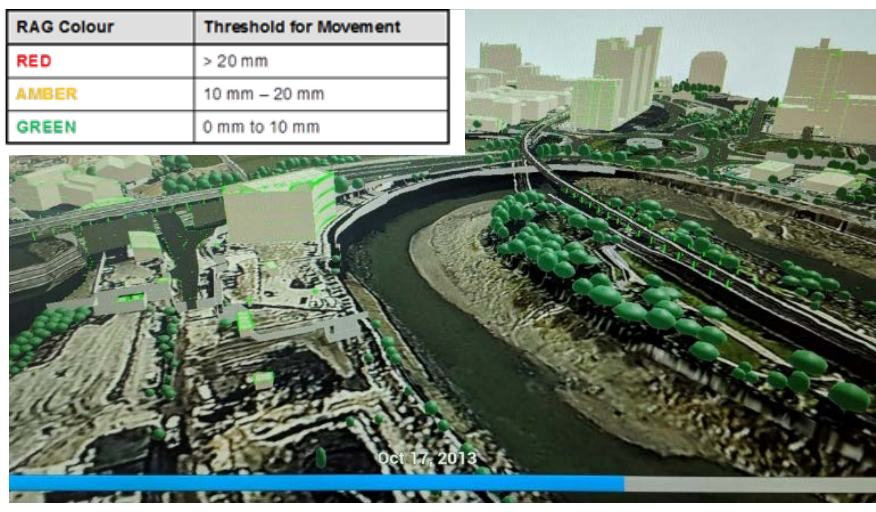

Figure 6: Measurements on 28th May 2014, showing the Red threshold being exceeded

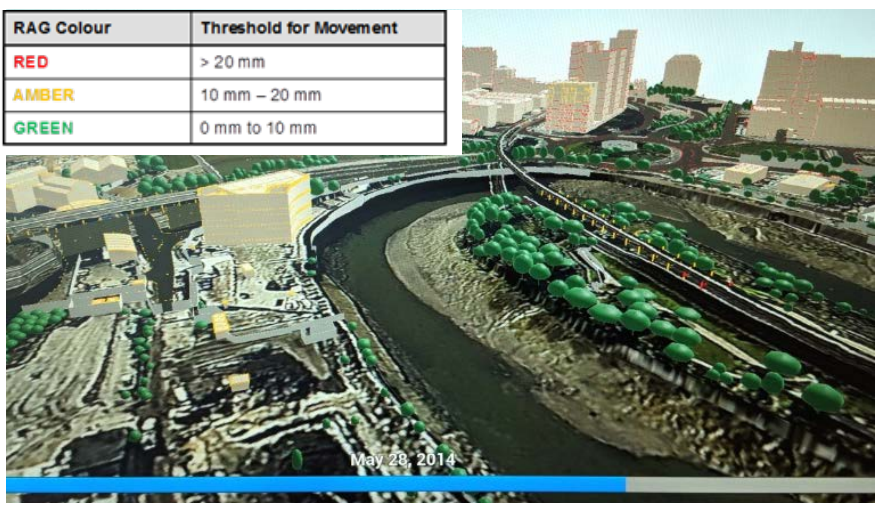

With regards of EDF case study at Hinkley Point, the three lifecycle stages of a Nuclear Power station were monitored for Structural Health: Construction (Hinkley Point C-HPC), Operations (Hinkley Point B-HPB) and decommissioning (Hinkley Point A-HPA).

S-SHM ${ }^{\mathrm{TM}}$ analysis was performed over the structures of HPA and $\mathrm{B}$ showing overall stability of the structures and some subsidence developing towards HPA caused by dewatering activities occurring on HPC construction phase. However, while these observations could not be validated due to the unavailability of local onsite measurement data, the overall S- 
SHM method does show much promise for surveillance of large infrastructure assets such as nuclear power stations.

Figure 7: S-SHM Measurements Hinkley site (EDF)

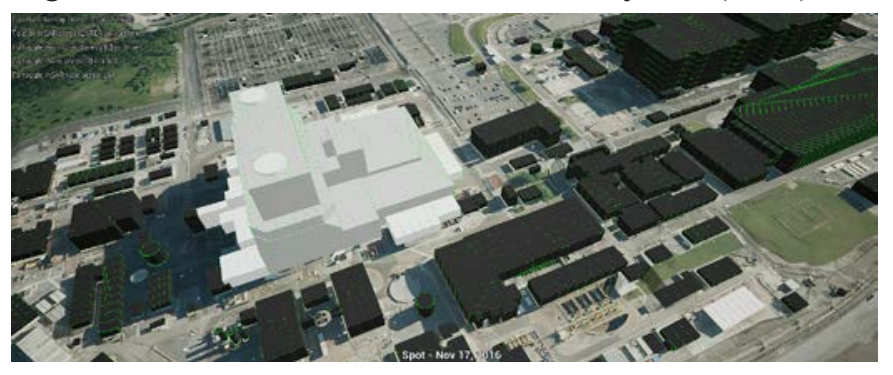

\section{S-SHM ${ }^{\mathrm{TM}}$ Scalability}

S-SHM ${ }^{\mathrm{TM}}$ is scalable and can be updated to include additional structural tests such as:

- Uniform raising/lowering of particular structures or single faces

- Very strong uptrend of measurements with respect to its baseline.

- Up bias of measurements with respect to its baseline.

- Stable measurements with respect to its baseline.

- Down bias with respect to its baseline.

- Very strong downtrend of measurements with respect to its baseline

- Oscillations with respect to a baseline.

One of the key conclusions of the work has been that remotely sensed structural health monitoring is feasible. It provides high quality and reliable outputs on structural health as well as surrounding regions. It can be implemented without the need for in-situ instrumentation. This is particularly useful for difficult to access and hazardous environments such as nuclear power stations, chemical plants, offshore locations and other extreme environments.

\section{Further Implementations}

S-SHM ${ }^{\mathrm{TM}}$ allows asset condition analysis but assets cannot be studied in isolation to fully assess their resilience, the surrounding environment has to be part of the condition analysis of this symbiotic relationship.

${\mathrm{S}-\mathrm{SHM}^{\mathrm{TM}}}^{\mathrm{is}}$ being deployed also as part of the EC H2020 project FORESEE, where the structural health of multiple assets in two different sites in Europe will be studied within their natural environment looking at the impact caused by natural hazards: landslides, flooding and seismicity.
Infrastructure resilience will be studied from different scales with satellite InSAR surface movement data, from regional to asset level, identifying and prioritising vulnerable areas to climate-related hazards

Once areas vulnerable to climate-linked risks have been identified, S-SHM ${ }^{\mathrm{TM}}$ will be implemented in several assets providing structural health condition, evolution and asset condition predictions

FORESEE aims to monitor key assets of large transport infrastructures located in risk prone areas using a multiscale analysis approach by:

- Providing a strategy to acquire satellite derived ground and asset displacement data at different scales and resolutions to analyse the dynamics of the terrain, such us landslides, seismicity or other movement processes as well as the effects of flooding and their potential effects in infrastructures in large transportation corridors.

- Developing a GIS/BIM system integrating different sources of data to intelligently relate the cause/effect of extreme events to infrastructure damage.

- Identifying and prioritising areas vulnerable to climate-related hazards using a GIS based multicriteria decision-making tool taking into account the social impact and the economic disruption by the incorporation of traffic and population demographics.

- Using ground motion data derived from satellites in order to constrain parameters in a condition prediction model, which can then be deployed in areas without satellite ground motion data.

- Developing a BIM based alerting Situational Awareness platform.

Figure 8: FORESEE overall approach

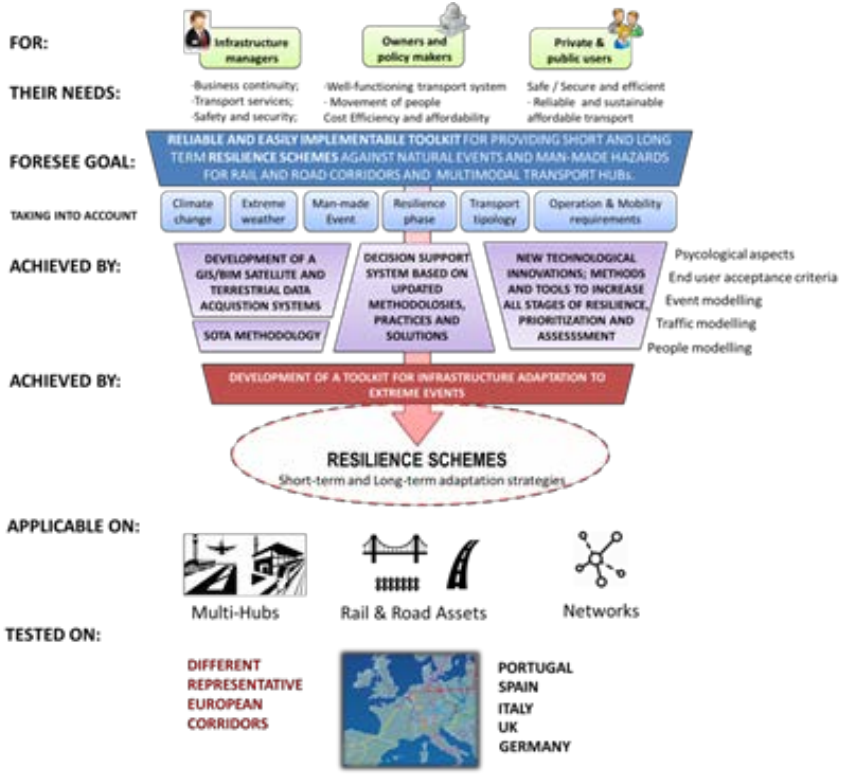


$\mathrm{S}^{-\mathrm{SHM}^{\mathrm{TM}}}$ is also included in a Critical National Infrastructure Integrated Applications Project (CNI IAP). This project combines Space-based and in-situ data to develop services to support resilient and sustainable Critical Infrastructure by offering increased protective measures for high value critical energy sites involved in the processing, refining, storage and distribution of oil, gas and electricity (from nuclear). The project involves:

- $\mathrm{S}-\mathrm{SHM}^{\mathrm{TM}}$ - Asset monitoring via wide area noninvasive ground deformation analysis via satellite InSAR technology (as described in this paper)

- In-situ sensors providing continuous real time pipeline flow rate monitoring and leak detection

- Monitoring methane emissions from satellite

\section{Acknowledgements}

To TfL and EDF for their continuous support in the implementation of new technologies for current infrastructure challenges and the Digital transformation of Infrastructures.

To Innovate UK whose grant funding was used in the development of S-SHM.

To Telespazio's project partners in the Innovate UK activity: STLTech, TWI, and in particular ThinkLab (University of Salford) who were responsible for the 3D visualisations shown.

\section{References}

O’Brien J (2017) Maintenance metrics: planned maintenance percentage. https://www.fiixsoftware.com/blog/advancedcmms-metrics-planned-maintenance-percentage/

Tongue A Current Challenges for Structural Health Monitoring. http://www.sensuron.com/industrynews/structural-health-monitoring-systems/

Shawn B (2005) Practical Issues in Real-World Implementation of Structural Health Monitoring Systems, Shawn J. Beard et al, SPIE Smart Structures and Materials + Nondestructive Evaluation and Health Monitoring. https://apps.dtic.mil/dtic/tr/fulltext/u2/a442245.pdf 